\title{
Development of Retinal Amacrine Cells and Their Dendritic Stratification
}

\author{
Revathi Balasubramanian · Lin Gan
}

Published online: 5 July 2014

(C) Springer Science + Business Media New York 2014

\begin{abstract}
The mammalian retina contains multiple neurons, each of which contributes differentially to visual processing. Of these retinal neurons, amacrine cells have recently come to prime light since they facilitate majority of visual processing that takes place in the retina. Amacrine cells are also the most diverse group of neurons in the retina, classified majorly based on the neurotransmitter type they express and morphology of their dendritic arbors. Currently, little is known about the molecular basis contributing to this diversity during development. Amacrine cells also contribute to most of the synapses in the inner plexiform layer and mediate visual information input from bipolar cells onto retinal ganglion cells. In this review, we will describe the current understanding of amacrine cell and cell subtype development. Furthermore, we will address the molecular basis of retinal lamination at the inner plexiform layer. Overall, our review will provide a developmental perspective of amacrine cell subtype classification and their dendritic stratification.
\end{abstract}

Keywords Retina - Amacrine cells - Retinogenesis ·

Transcription factors $\cdot$ Dendritic stratification

\section{Introduction}

The neural retina is broadly categorized into seven distinct cell types (Fig. 1a): retinal ganglion cells, amacrine cells, bipolar

\footnotetext{
R. Balasubramanian · L. Gan

Department of Ophthalmology and Flaum Eye Institute,

University of Rochester, Rochester, NY 14642, USA

R. Balasubramanian · L. Gan ( $\square)$

Department of Neurobiology and Anatomy, University of

Rochester, Rochester, NY 14642, USA

e-mail: lin_gan@urmc.rochester.edu
}

cells, Müller glial cells, horizontal cells, and rod and cone photoreceptor cells. Anatomically, the mammalian retina is composed of three layers of nerve cell bodies (ganglion cell layer, inner nuclear layer, and outer nuclear layer) and two layers of synapses (inner plexiform layer and outer plexiform layer). The ganglion cell layer (GCL) contains cell bodies of ganglion cells and displaced amacrine cells. The inner nuclear layer (INL) contains cell bodies of amacrine cells, Müller glial cells, horizontal cells, and bipolar cells. The outer nuclear layer (ONL) contains cell bodies of cone and rod photoreceptors. Connections between bipolar cells, horizontal cells, and cone and rod photoreceptors occur in the outer plexiform layer (OPL), while that between bipolar cells, amacrine cells, and ganglion cells occur in the inner plexiform layer (IPL) [1, 2•]. The diversity of retinal neurons goes further beyond retinal cell classification with each retinal cell type being further classified into many subtypes [3]. This neuronal diversity is brought about during retinogenesis by the changing competence of progenitor cells with time [4]. This leads to the specification and differentiation of different retinal cell classes and their subtypes and a distinction in their laminar stratification.

Recently, amacrine cells and their multitude of subtypes have been of particular interest, partly because amacrine cells display the highest degree of diversity in both morphology and function in the retina $\left[5,6^{\bullet}\right]$. So far, up to thirty distinct subpopulations of amacrine cells have been identified based on the neurotransmitter type they express, dendrite morphology, and/or functional characteristics [3, 5]. However, the list is still growing and the precise number of subtypes of amacrine cells is currently unknown.

Subtype Classification of Amacrine Cells

Amacrine cells have been categorized into at least 33 different subtypes based on their dendritic morphology and 

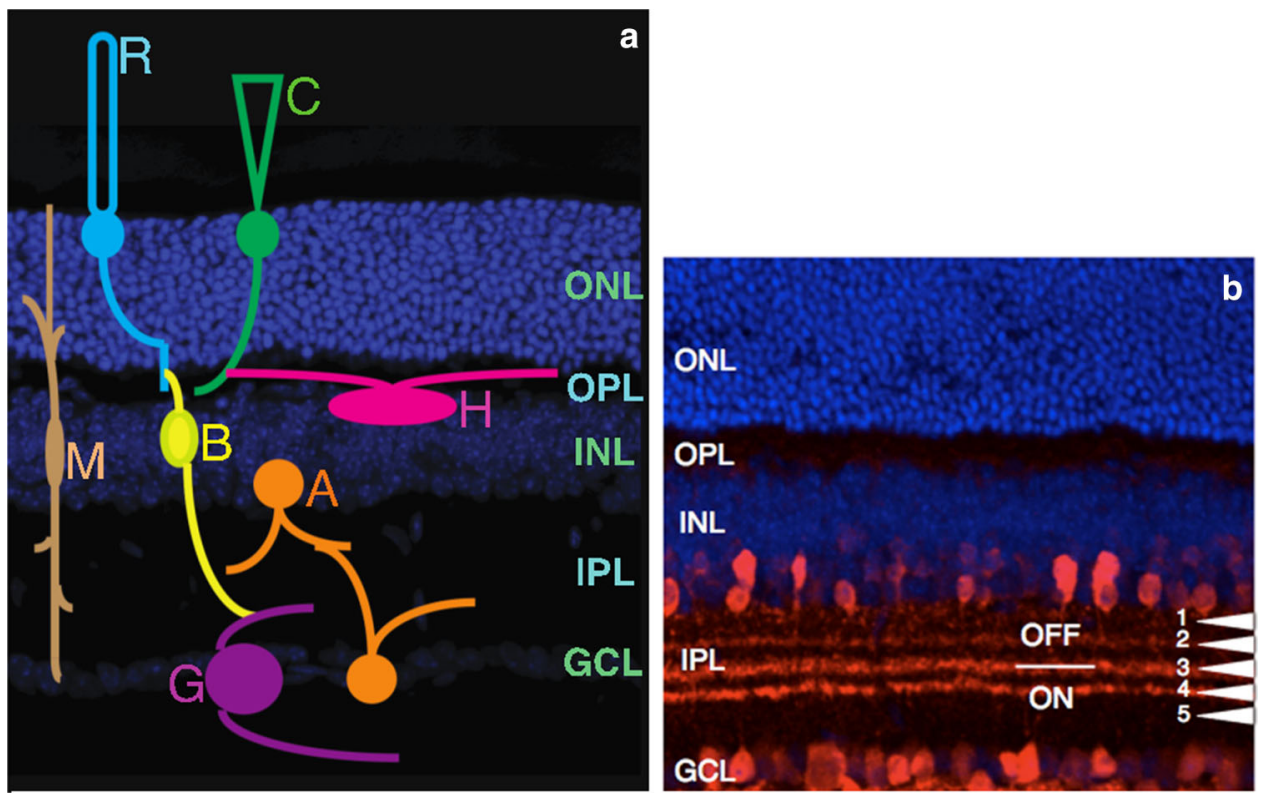

Fig. 1 Diagrammatic representation of retinal neurons in distinct retinal layers. a Localization of rod and cone photoreceptors and their cell bodies in the ONL; horizontal cells, bipolar cells, amacrine cells, and Müller glial cells in the INL; and retinal ganglion cells and displaced amacrine cells in the GCL. b Anti-calretinin staining (red) of the IPL marks 4 of the 5 sublaminas. S1 and S2 are in the OFF lamina, S4 and S5 are in the ON lamina, and S3 is a watershed region

stratification. Pioneering studies by Masland, Vaney, Wassle, and others identified these subtypes of amacrine cells in the rodent retina [6-15]. Based on how proliferative their dendritic arbors are, they are categorized as narrow-field amacrine cells (dendritic arbor of around $70 \mu \mathrm{m}$ in diameter), medium-field amacrine cells (dendritic arbor of around $170 \mu \mathrm{m}$ in diameter), and wide-field amacrine cells (dendritic arbor of about $350 \mu \mathrm{m}$ in diameter). Amacrine cells contribute toward majority of synapses in the IPL. Based on axonal projections of OFF and ON bipolar cells, the IPL is divided into an OFF lamina and an ON lamina, respectively. These laminas are further divided into five parallel sublaminas (S1-S5) based on the dendritic stratification of subtypes of amacrine cells [1, 2•] (Fig. 1b).

Based on their ramification in the IPL, amacrine cells are classified into various subtypes. The details of individual subtypes categorized based on morphology will not be discussed here, an extensive review being available in $[6 \bullet, 10 \bullet]$.

Amacrine cells are inhibitory interneurons that project their dendritic arborization to the IPL where they intercept retinal ganglion cells and/or bipolar cells. Based on the inhibitory neurotransmitter type they express, amacrine cells are classified into two major subpopulations: GABAergic and glycinergic types [6, 10]. A small population of between the ON and OFF lamina. Sections are counterstained with DAPI (blue). $R$ rod photoreceptor, $C$ cone photoreceptor, $M$ Müller glial cell, $B$ bipolar cell, $A$ amacrine cell, $H$ horizontal cell, $G$ retinal ganglion cell, $O N L$ outer nuclear layer, $O P L$ outer plexiform layer, $I N L$ inner nuclear layer, $I P L$ inner plexiform layer, $G C L$ ganglion cell layer (Color figure online)

amacrine cells has also been found to be neither GABAergic nor glycinergic [16•]. GABAergic amacrine cells are distributed in both the GCL as well as the INL, have mostly wide-field dendritic arbors, and can be further classified by their expression of neurotransmitters and neuropeptides such as choline acetyl transferase (ChAT), neuropeptide Y (NPY), tyrosine hydroxylase (TH), or nitric oxide synthase (NOS) [12•, 17-21]. Glycinergic amacrine cells are largely restricted to the INL, have mostly narrowfield dendritic arbors, and can be further identified by the expression of Disabled-1 (Dabl), parvalbumin (PV), or vesicular glutamate transporter type 3 (VGlut3) [12•, 13, 14, 22-25]. Developmentally, GABAergic amacrine cells are born first with their developmental peak at about E14, while glycinergic amacrine cells are born later with peak of birth at about P0 [26].

Neurotransmitters and neuropeptide markers are useful in distinguishing between the different cell subtypes of amacrine cells. Calcium binding proteins such as calbindin and calretinin have also been used to characterize amacrine cells and specifically to visualize their stratification in the IPL. Depending on the species, calretinin, for instance, marks a subclass of amacrine cells and retinal ganglion cells. It also labels three prominent strata of the IPL-S2, S3, and S4, with weak expression also seen in S1 (Fig. 1b). Calbindin marks horizontal cells, some amacrine cells and 
ganglion cells and also labels $\mathrm{S} 2, \mathrm{~S} 3$, and $\mathrm{S} 4$ strata of the IPL [12•].

A significant percentage of GABAergic amacrine cells that express ChAT (otherwise known as starburst amacrine cells) are present both in the INL and GCL. Cholinergic amacrine cells from the INL ramify in the OFF sublamina $\mathrm{S} 2$ of the IPL, while those from the GCL ramify in the ON sublamina S4 [12•]. Developmentally, cholinergic amacrine cells are among the first to be specified at around E11 [26•]. Cholinergic amacrine cells in the GCL are born first followed closely by cholinergic amacrine cells of the INL, lending credence to a theory that the position of soma in the retina might correlate with birthdates. Starburst amacrine cells are among the most extensively studied subset of amacrine cells, and it is well known that starburst amacrine cells play a major role in modulating the response of direction sensitive retinal ganglion cells and hence contribute to direction selectivity and detection of directional motion (reviewed in [27]).

NPY is expressed in a population of GABAergic amacrine cells that are located in both the INL and GCL. NPY cells in the INL project their dendrites mostly to the OFF sublamina S1, while those in the INL, to the ON sublamina S5 [18, 20]. A small population of NPY cells also expresses $\mathrm{TH}$ and projects to S3 sublamina. Interestingly, Voinescu et al. [26•] found coincidental expression of NPY in all cholinergic amacrine cells at P7. As development proceeds, NPY expression in cholinergic amacrine cells reduces. This indicates that not only do progenitor cells change their competence to produce different retinal types, but the same mechanism might be at play in specifying retinal cell subtypes as well. Functionally, NPY seems to have a role in mediating low-frequency spatial processing (large spatial patterns) by retinal ganglion cells [28].

Almost all dopaminergic amacrine cells (TH expressing) are GABAergic and are located at the boundary of INL and IPL and ramify in S1 lamina of the IPL. Studies that use a TH promoter-driven RFP/GFP line identify a group of GABAergic catecholaminergic cells (Type II cells) that do not contain dopamine and specifically stratify in S3 [21, 29]. It is possible these cells express NPY as reported by Oh et al. [18] who found a subpopulation of NPY cells that were immunoreactive for TH. Functionally, dopaminergic amacrine cells modulate light adaptation and circadian rhythm.

Another group of GABAergic amacrine cells express neuronal NOS. NOS-expressing amacrine cells have been categorized further based on the size of their soma and dendritic arborization. Types I and II are situated in the INL and ramify in S1 and mainly in S3. The displaced NOS amacrine cells in the GCL ramify mainly in S4 and S5 [17, 30]. NOS amacrine cells usually intercept and interact with other amacrine cells in the IPL, but some synapse with retinal ganglion cells as well. Some cells also mediate input from bipolar cells projecting to the ON sublamina. Current studies seem to suggest that NO amacrine cells might play a role in visual adaptation since NO synthesis is largely dependent on stimulation by light [19, 30].

Glycinergic amacrine cells are not extensively characterized due to the lack of an extensive marker set to label the different subtypes of glycinergic cells. Glycinergic amacrine cells as a whole are marked by glycine transporter (GlyT1) [14]. One well-characterized subset of glycinergic amacrine cells is the AII amacrine cells [9, 24, 31]. AII amacrine cells are present primarily in the INL and are rarely found in the GCL. They can be marked by cytoplasmic protein DAB1, which is part of the reelin pathway that controls cell positioning during development in parts of the central nervous system. DAB1 is visibly expressed in AII glycinergic amacrine cells as early as P7 in the mouse retina [22]. PV is another marker for AII amacrine cells as PV-expressing cells are morphologically similar to AII amacrine cells and also co-express DAB1 $[12,22,23]$. In the adult mouse retina, the AII amacrine cells ramify primarily in the ON S5 sublamina. DAB1 immunoreactivity is also discernible as thick lobules at the distal part of the OFF sublamina. Studies have suggested that these lobules are sites of inhibitory interaction between AII amacrine cells, OFF bipolar cells, and OFF retinal ganglion cells. The inhibitory interaction between AII amacrine cells and OFF bipolar cells may be important for visual responses at high spatial frequencies and/or detecting motion of varying spatiotemporal contrasts [31].

Vesicular glutamate transporter type 3 (VGlut3) is expressed in a subset of glycinergic amacrine cells in the INL. VGlut3 amacrine cells ramify in the ON and OFF sublaminas (S2 and S3), filling in the gaps between the three calretinin bands and seem to make connections with OFF cone bipolar cells at S2, ON cone bipolar cells at S3, and rod bipolar cells at S4/S5 [13, 32]. VGlut3 cells seem to receive inputs primarily from amacrine cells and hence are thought to be involved in amacrine-to-amacrine cell crossover inhibition. A population of VGlut3 cells expresses circadian clock gene Period 1 (Perl) suggesting that this population might have a role in generating circadian rhythms [25].

There is also a small population of amacrine cells that are neither GABAergic nor glycinergic ( $\mathrm{nGnG})$. Roughly $85 \%$ of amacrine cells are accounted for, by staining with glutamic acid decarboxylase 65/67 (GAD65/67) for GABAergic cells or GlyT1 for glycinergic amacrine cells. The remaining $\sim 15 \%$ of amacrine cells express neither. The neurotransmitter type(s) expressed by these amacrine cells is currently unknown. $\mathrm{nGnG}$ amacrine cells ramify primarily to the OFF lamina $(\mathrm{S} 1-\mathrm{S} 3)$ of the IPL with their dendritic arborization being morphologically narrow field. 
Developmentally, these cells are born after glycinergic cells during the first few postnatal days [16•].

Amacrine Cells Contribute to the Majority of Synapses Formed in the IPL

Amacrine cells and bipolar cells project presynaptic dendrites to the IPL where they synapse with retinal ganglion cells. Various aspects of visual information encoded by retinal neurons are transmitted to retinal ganglion cells via specific synapses in the IPL, and hence it is pertinent that the specification of IPL occurs in a well-directed and timely manner. Yamagata et al. [33] first identified and elucidated the role of two homologous transmembrane immunoglobulin superfamily molecules (sidekick family) - Sdk1 and $S d k 2$-in retinal lamination of the chick retina. They found that $S d k 1$ and $S d k 2$ are expressed in mutually exclusive populations of retinal neurons in the INL and GCL. While $S d k 2$ expression is greater in sublamina S2 of the IPL and weaker in S4, the opposite is true of $S d k 1$. Over-expression of Sdks in cells that are devoid of it, rerouted these cells to the Sdk-positive layer without a change in cell identity. This suggests that Sdks play an important role in promoting laminar synaptic formation by promoting synapse formation between pre- and post-synaptic Sdk-positive neurons. Further, they have identified the presence of related adhesion molecules, Dscam and DscamL, in exclusive layers of the IPL. Dscam expression is restricted to S5, while DscamL is expressed in S1, S2, and S4 [34]. Dscam is also localized to the somata of the TH- and NOS-expressing amacrine cells. Loss- and gainof-function studies demonstrated that the Dscam adhesion molecules along with Sdks regulate the development of sublaminar stratification across the IPL [34]. Dscams are also shown to influence mosaic spacing by promoting selfavoidance between different types of amacrine cells in the INL, which is essential to preserve functionality of amacrine neurons [35]. Another class of transmembrane signaling molecules-semaphorins, signaling via their receptors-plexins, display complementary expression patterns across the IPL and regulate retinal lamination during development $[36,37]$. In Sema5A and Sema5B double null mutants, TH- and VGlut3-expressing amacrine cells display gross dendritic mistargeting in the IPL. Other amacrine types like AII amacrine cells, cholinergic amacrine cells, calretinin, and calbindin-expressing amacrine cells display prominent ectopic projections of dendrites toward the OPL. Double null mutants of receptors PlexAl and PlexA3 phenocopy the anomalies in stratification displayed by Sema5A and Sema5B double mutants [37]. Sema6A ligand and PlexA4 receptor combination are also expressed in a complimentary manner through IPL development. PlexA4 is expressed in S1 and S2, and Sema6A is expressed in S3, S4, and S5 laminas of the IPL. PlexA4 mutants display aberrant dendritic projections of $\mathrm{TH}-$ expressing amacrine cells, calbindin-expressing amacrine cells, and intrinsically photosensitive retinal ganglion cells, with Sema6A-null mutants phenocopying the aberrant dendritic stratification, suggesting that Sema6A acts a functional ligand for PlexA4 in regulating retinal lamination at the IPL [36]. Taken together, transmembrane guidance molecules have been shown to regulate neurite stratification and retinal lamination at the IPL by influencing the development of dendritic arborization of amacrine cells among others. Recent studies in Zebrafish have shown the role of transcription factor $\operatorname{Irx} 7$ in regulating retinal lamination by affecting neurite outgrowth [38]. Another factor namely PTEN phosphatase also mediates the mosaic spacing, fasciculation, and dendritic arborization of TH amacrine cells and of horizontal cells, likely in a Dscam/Sema-independent manner [39]. Although we currently understand the role of these signaling molecules in IPL development, we are yet to assimilate the full network of molecules upstream and downstream of the signaling factors that play into this regulation.

Transcription Factors Influencing the Development of Amacrine Cell Types and Subtypes

During development, amacrine cells are specified early along with retinal ganglion cells, cone photoreceptors, and horizontal cells. Bipolar cells, rod photoreceptors, and Müller glial cells are specified later. In mouse retina, amacrine cells are born between embryonic day 8 (E8) and postnatal day 5 (P5), with the peak of competence between E14 and E16 $[4,26]$. The competence of the early retinal progenitor pool changes with time and the expression of intrinsic factors such as transcription factors are instrumental in specifying the identity of the retinal cell class as a whole and of individual cell subtypes. Here, we discuss some of the molecular factors governing the differentiation and specification of amacrine cells and their subtypes.

Early factors such as Pax6, Sox2, Bclaf1 influence the competence of retinal progenitors and significantly impact the development of amacrine cells from the early progenitor pool. Initial studies on Pax6 showed that a conditional deletion of Pax6 in the eye resulted in the production of only amacrine cells with a loss of most other neuron types and is hence necessary for the maintenance of progenitors in their multi-potent state [40, 41]. Furthermore, all amacrine cells born in the Pax6 mutant were identified as GABAergic, hence further restricting the progenitor fate choice to a non-glycineric amacrine cell type. Bclafl has been recently identified as a factor that could potentially act along with Pax6 in the differentiation of early retinal neuron population [42]. Sox2 is expressed in Müller glial 
cells and the cholinergic subset of amacrine cells in the adult retina. During development, over-expression of Sox2 in explant cultures biases progenitor cells to an amacrine cell fate [43].

Conditional deletion and over-expression studies of Foxn 4 show that it is necessary and sufficient for the generation of amacrine and horizontal cells. Foxn 4 deletion greatly attenuated amacrine cell numbers, while an overexpression of Foxn4 in progenitors greatly facilitated amacrine cell fate (both glycinergic and GABAergic) at the expense of rod photoreceptors [44, 45•]. Recent studies in the chick retina have shown that Foxn4 expression might be in part regulated by Meis 1 , which regulates the activity of a non-coding DNA fragment upstream of the Foxn4 transcription start site [46]. Foxn4 also interacts with its downstream targets-Neurod4 (previously Math3) and Neurodl, and regulates the differentiation of multi-potent progenitors into amacrine cells [44]. A loss of Neurod4 or Neurodl alone does not affect amacrine cell numbers, whereas a double mutant of Neurodl and Neurod4 completely abolished amacrine cell generation. An overexpression of Neurod4 or Neurodl alone does not restore amacrine cell numbers, but addition of Pax6 to either Neurodl or Neurod4 is sufficient to generate amacrine cells [47]. This signifies that Neurod4 and Neurodl have mutually redundant functions in amacrine cell genesis, and act downstream of Foxn4 and Pax6. While they are together essential for the generation of amacrine cells, they are not sufficient for amacrine cell genesis. Another factor acting downstream of Foxn4 is Ptfla, the loss of which biases precursors of amacrine and horizontal cells into retinal ganglion cell fate leading to greatly reduced amacrine cell numbers [48, 49]. Gain-of-function and loss-offunction studies in the Xenopus retina indicate that Ptfla might be particularly essential for the development of GABAergic fate in amacrine cells [50].

Several transcription factors have been studied for their role in amacrine subtype specification. Barhl2 is one such factor, which is expressed in bipolar cells, amacrine cells, and retinal ganglion cells. A forced expression study of Barhl2 suggested that it might be an important regulator of the glycinergic subtype development [51]. Loss-of-function studies have shown that Barhl2 attenuates both glycinergic and GABAergic amacrine cell numbers with a concomitant increase in cholinergic amacrine cells [52]. Is $l 1$ has been extensively studied for its role in cholinergic amacrine cell development. Isll is expressed in retinal ganglion cells, cholinergic amacrine cells, and bipolar cell subtypes [53]. Loss of $I s l 1$ results in the loss of about $93 \%$ of cholinergic amacrine cells and a concomitant disruption of the S2 and S4 stratification in the IPL [54]. Bhlhb5 is expressed in GABAergic amacrine cells and cone bipolar cells in the adult. Loss of Bhlhb5 expression results in a loss of about a third of GABAergic cells. TH cells seem to be the most affected one with about a $90 \%$ decrease in Bhlhb5 mutants [55]. Lineage tracing studies have shown that Bhlhb5-expressing progenitors give rise to cholinergic, glycinergic, and GABAergic amacrine cell fates. The expression in the adult in cholinergic and glycinergic amacrine cells is downregulated but is retained in GABAergic amacrine cell subtypes [56]. Lmo4 is expressed in a subset of GABAergic amacrine cells, and a conditional deletion of Lmo4 leads to a reduction in the number of Bhlhb5-expressing GABAergic amacrine cells [57]. Analysis of specific expression of individual members of the Neurod 1 transcription factor family showed the expression of Neurod2 specifically in Dab1-expressing AII amacrine cells. Loss of Neurod2 results in a partial loss of AII amacrine cells [58]. Neurod6 expression in adult amacrine neurons was also identified and is expressed mostly in nGnG amacrine cells [16, 58]. Loss of Neurod6 results in an increase in the number of glycinergic amacrine cells at the expense of $\mathrm{nGnG}$ amacrine cells [16॰]. Interplay between Neurod6 and Satb2/Ebf3-expressing amacrine cells regulates the decision of the progenitor cells to differentiate into glycinergic amacrine cells versus the $\mathrm{nGnG}$ fate. Ebf class transcription factors have been studied for their role in the differentiation of AII class amacrine cells. Gain- and loss-of-function studies indicate that Ebf class transcription factors are both necessary and sufficient for specifying AII amacrine subtypes [59].

\section{What Remains Unknown?}

We are currently aware of the presence of the many subtypes of amacrine cells but unclear on their individual functions in processing visual information. We have elucidated some of the transcription factors that play an important role in the development of amacrine cells class as a whole, but our understanding of how these factors come together or compete with other factors responsible for specifying other retinal cell types is currently limited. Molecular mechanisms governing the development of individual amacrine cells subtypes and their specific laminar stratification are also currently limited. Identifying unique regulatory factor or a unique combination of factors responsible for amacrine subtype specification will be useful for answering some of these questions. We are now beginning to understand the molecular basis of laminar stratification and mosaic tiling of amacrine cells, but are yet to determine the influence of the complete synaptic laminar network involving retinal ganglion cells, amacrine cells, and bipolar cells on retinal function. Identifying the developmental factors responsible for laminar stratification has been immensely useful, allowing us to mark cells and their synaptic contributions to the IPL. More work in this 
direction will help put together a developmental framework which will ultimately provide us with a deeper understanding of amacrine cells and their contribution to visual information processing.

Disclosure Revathi Balasubramanian and Lin Gan have declared no conflicts of interest.

Human and Animal Rights and Informed Consent This article does not contain any studies with human or animal subjects performed by any of the authors.

\section{References}

Papers of particular interest, published recently, have been highlighted as:

- Of importance

1. Masland RH. The neuronal organization of the retina. Neuron. 2012;76(2):266-80.

2. - Masland RH. The fundamental plan of the retina. Nat Neurosci. 2001;4(9):877-86. This article describes the diversity of retinal neurons and its association with function.

3. Masland RH. Neuronal diversity in the retina. Curr Opin Neurobiol. 2001;11(4):431-6.

4. Livesey FJ, Cepko CL. Vertebrate neural cell-fate determination: lessons from the retina. Nat Rev Neurosci. 2001;2(2):109-18.

5. Masland RH. The tasks of amacrine cells. Vis Neurosci. 2012;29(1):3-9.

6. - MacNeil MA, Masland RH. Extreme diversity among amacrine cells: implications for function. Neuron. 1998;20(5):971-82. This article describes the morphological diversity among amacrine cells and explores the functions of amacrine interneurons.

7. Vaney DI. Morphological identification of serotonin-accumulating neurons in the living retina. Science. 1986;233(4762):444-6.

8. Vaney DI. Territorial organization of direction-selective ganglion cells in rabbit retina. J Neurosci. 1994;14(11 Pt 1):6301-16.

9. Vaney DI, Gynther IC, Young HM. Rod-signal interneurons in the rabbit retina: 2. AII amacrine cells. J Comp Neurol. 1991;310(2):154-69.

10. - MacNeil MA, et al. The shapes and numbers of amacrine cells: matching of photofilled with golgi-stained cells in the rabbit retina and comparison with other mammalian species. J Comp Neurol. 1999;413(2):305-26. This article provides a thorough classification of amacrine cells based on their dendritic stratification.

11. Wassle H, Grunert U, Rohrenbeck J. Immunocytochemical staining of AII-amacrine cells in the rat retina with antibodies against parvalbumin. J Comp Neurol. 1993;332(4):407-20.

12. - Haverkamp S, Wassle H. Immunocytochemical analysis of the mouse retina. J Comp Neurol. 2000;424(1):1-23. Using immunohistochemistry, this report classifies amacrine interneurons based on neurotransmitters or neuropeptides they express.

13. Haverkamp S, Wassle H. Characterization of an amacrine cell type of the mammalian retina immunoreactive for vesicular glutamate transporter 3. J Comp Neurol. 2004;468(2):251-63.

14. Menger N, Pow DV, Wassle H. Glycinergic amacrine cells of the rat retina. J Comp Neurol. 1998;401(1):34-46.

15. Strettoi E, Masland RH. The organization of the inner nuclear layer of the rabbit retina. J Neurosci. 1995;15(1 Pt 2):875-88.
16. - Kay JN, et al. Neurod6 expression defines new retinal amacrine cell subtypes and regulates their fate. Nat Neurosci. 2011;14(8):965-72. An excellent reference describing the development and characterization of $n G n G$ (neither glycinergic nor GABAergic) amacrine cells.

17. Haverkamp S, Kolb H, Cuenca N. Morphological and neurochemical diversity of neuronal nitric oxide synthase-positive amacrine cells in the turtle retina. Cell Tissue Res. 2000;302(1):11-9.

18. Oh SJ, et al. Distribution and synaptic connectivity of neuropeptide Y-immunoreactive amacrine cells in the rat retina. J Comp Neurol. 2002;446(3):219-34.

19. Pang JJ, Gao F, Wu SM. Light responses and morphology of bNOS-immunoreactive neurons in the mouse retina. J Comp Neurol. 2010;518(13):2456-74.

20. Sinclair JR, Nirenberg S. Characterization of neuropeptide Y-expressing cells in the mouse retina using immunohistochemical and transgenic techniques. J Comp Neurol. 2001;432(3):296-306.

21. Eglen SJ, et al. Dopaminergic amacrine cells in the inner nuclear layer and ganglion cell layer comprise a single functional retinal mosaic. J Comp Neurol. 2003;466(3):343-55.

22. Rice DS, Curran T. Disabled-1 is expressed in type AII amacrine cells in the mouse retina. J Comp Neurol. 2000;424(2):327-38.

23. Yeo JY, Lee ES, Jeon CJ. Parvalbumin-immunoreactive neurons in the inner nuclear layer of zebrafish retina. Exp Eye Res. 2009;88(3):553-60.

24. Lee EJ, et al. AII amacrine cells in the distal inner nuclear layer of the mouse retina. J Comp Neurol. 2006;494(4):651-62.

25. Johnson J, et al. Vesicular glutamate transporter 3 expression identifies glutamatergic amacrine cells in the rodent retina. J Comp Neurol. 2004;477(4):386-98.

26. - Voinescu PE, Kay JN, Sanes JR. Birthdays of retinal amacrine cell subtypes are systematically related to their molecular identity and soma position. J Comp Neurol. 2009;517(5):737-50. This report details the developmental time line of a number of amacrine cell subtypes.

27. Taylor WR, Smith RG. The role of starburst amacrine cells in visual signal processing. Vis Neurosci. 2012;29(1):73-81.

28. Sinclair JR, Jacobs AL, Nirenberg S. Selective ablation of a class of amacrine cells alters spatial processing in the retina. J Neurosci. 2004;24(6): 1459-67.

29. Meng S, et al. Targeting retinal dopaminergic neurons in tyrosine hydroxylase-driven green fluorescent protein transgenic zebrafish. Mol Vis. 2008;14:2475-83.

30. Vielma AH, Retamal MA, Schmachtenberg O. Nitric oxide signaling in the retina: what have we learned in two decades? Brain Res. 2012;1430:112-25.

31. Demb JB, Singer JH. Intrinsic properties and functional circuitry of the AII amacrine cell. Vis Neurosci. 2012;29(1):51-60.

32. Grimes WN, et al. Genetic targeting and physiological features of VGLUT3+ amacrine cells. Vis Neurosci. 2011;28(5):381-92.

33. Yamagata M, Weiner JA, Sanes JR. Sidekicks: synaptic adhesion molecules that promote lamina-specific connectivity in the retina. Cell. 2002;110(5):649-60.

34. Yamagata M, Sanes JR. Dscam and Sidekick proteins direct lamina-specific synaptic connections in vertebrate retina. Nature. 2008;451(7177):465-9.

35. Fuerst PG, et al. Neurite arborization and mosaic spacing in the mouse retina require DSCAM. Nature. 2008;451(7177):470-4.

36. Matsuoka RL, et al. Transmembrane semaphorin signalling controls laminar stratification in the mammalian retina. Nature. 2011;470(7333):259-63.

37. Matsuoka RL, et al. Class 5 transmembrane semaphorins control selective mammalian retinal lamination and function. Neuron. 2011;71(3):460-73. 
38. Zhang Y, et al. The expression of irx7 in the inner nuclear layer of zebrafish retina is essential for a proper retinal development and lamination. PLoS ONE. 2012;7(4):e36145.

39. Cantrup R, et al. Cell-type specific roles for PTEN in establishing a functional retinal architecture. PLoS ONE. 2012;7(3):e32795.

40. Marquardt T, et al. Pax6 is required for the multipotent state of retinal progenitor cells. Cell. 2001;105(1):43-55.

41. Oron-Karni V, et al. Dual requirement for Pax6 in retinal progenitor cells. Development. 2008;135(24):4037-47.

42. Orieux $\mathrm{G}$, et al. Involvement of Bcl-2-associated transcription factor 1 in the differentiation of early-born retinal cells. J Neurosci. 2014;34(4):1530-41.

43. Lin YP, et al. Sox 2 plays a role in the induction of amacrine and Muller glial cells in mouse retinal progenitor cells. Invest Ophthalmol Vis Sci. 2009;50(1):68-74.

44. Li S, et al. Foxn 4 controls the genesis of amacrine and horizontal cells by retinal progenitors. Neuron. 2004;43(6):795-807.

45. - Ohsawa R, Kageyama R. Regulation of retinal cell fate specification by multiple transcription factors. Brain Res. 2008;1192:90-8. An excellent review that details the role of specific transcription factors during the development of amacrine cell types and subtypes.

46. Islam MM, et al. Meis1 regulates Foxn4 expression during retinal progenitor cell differentiation. Biol Open. 2013;2(11):1125-36.

47. Inoue $T$, et al. Math 3 and NeuroD regulate amacrine cell fate specification in the retina. Development. 2002;129(4):831-42.

48. Fujitani Y, et al. Ptf1 a determines horizontal and amacrine cell fates during mouse retinal development. Development. 2006;133(22): 4439-50.

49. Nakhai $\mathrm{H}$, et al. Ptfla is essential for the differentiation of GABAergic and glycinergic amacrine cells and horizontal cells in the mouse retina. Development. 2007;134(6):1151-60.
50. Dullin JP, et al. Ptf1a triggers GABAergic neuronal cell fates in the retina. BMC Dev Biol. 2007;7:110.

51. Mo Z, et al. Role of the Barhl2 homeobox gene in the specification of glycinergic amacrine cells. Development. 2004;131(7):1607-18.

52. Ding Q, et al. BARHL2 differentially regulates the development of retinal amacrine and ganglion neurons. $\mathrm{J}$ Neurosci. 2009;29(13):3992-4003.

53. Elshatory Y, et al. Expression of the LIM-homeodomain protein Isl1 in the developing and mature mouse retina. J Comp Neurol. 2007;503(1):182-97.

54. Elshatory Y, et al. Islet- 1 controls the differentiation of retinal bipolar and cholinergic amacrine cells. J Neurosci. 2007;27(46): 12707-20.

55. Feng L, et al. Requirement for Bhlhb5 in the specification of amacrine and cone bipolar subtypes in mouse retina. Development. 2006;133(24):4815-25.

56. Huang L, et al. Bhlhb5 is required for the subtype development of retinal amacrine and bipolar cells in mice. Dev Dyn. 2014;243(2):279-89.

57. Duquette PM, et al. Loss of LMO4 in the retina leads to reduction of GABAergic amacrine cells and functional deficits. PLOS ONE. 2010;5(10):e13232.

58. Cherry TJ, et al. NeuroD factors regulate cell fate and neurite stratification in the developing retina. J Neurosci. 2011;31(20): $7365-79$.

59. Jin K, et al. Early B-cell factors are required for specifying multiple retinal cell types and subtypes from postmitotic precursors. J Neurosci. 2010;30(36):11902-16. 